Post-print of: Macho-Stadler, Inés; Pérez-Castrillo, David; Wettstein, David "Dividends and weighted values in games with externalities" in International journal of game theory (Springer), Vol. 39, Num. 1 (2010) , p. 177-184, ISSN 0020-7276.

The final version is available at DOI: 10.1007/s00182-009-0207-0

\title{
Dividends and Weighted Values in Games with Externalities*
}

\author{
Inés Macho-Stadler ${ }^{\dagger} \quad$ David Pérez-Castrillo $\quad$ David Wettstein ${ }^{\S}$
}

August 28, 2009

\begin{abstract}
In this paper, we provide further support for the family of average values for games with externalities defined in Macho-Stadler et al. (2007). This is a large family of values that includes several recent proposals. We show that they can be constructed through the sharing of appropriately defined dividends. Furthermore, we show the flexibility of this approach by using it to generate non-symmetric values.
\end{abstract}

*We thank Guillaume Haeringer for his comments. We gratefully acknowledge the financial support from ECO2009-07616, 2009SGR-169, Consolider-Ingenio CSD2006-00016, Barcelona Economics-Xarxa CREA and ICREA Academia.

†Dep. of Economics \& CODE, Universitat Autònoma de Barcelona. Email: ines.macho@uab.es

$\ddagger$ Corresponding author. Dep. of Economics \& CODE, Universitat Autònoma de Barcelona, 08193 Bellaterra (Barcelona), Spain. Email: david.perez@uab.es. Ph.: (34) 935811405 and (34) 935812461. Fax: (34) 935812461.

$\S$ Dep. of Economics, Ben-Gurion University of the Negev. Email: wettstn@bgu.ac.il 


\section{Introduction}

In recent years there has been increasing interest in the construction and implementation of sharing rules for environments with externalities. Such environments encompass a large array of economic scenarios and arise whenever the benefits or costs to a group of agents depend on the coalitions formed by agents outside the group. Prominent examples are a market with several competing firms, countries negotiating on trade agreements and union-firm bargaining in a given industry.

One fruitful approach has been to proceed axiomatically and propose sharing methods (known as values) based on a collection of desirable properties. Some of the proposals extend the Shapley axioms (Shapley, 1953a), which are stated for games with no externalities. This is the direction taken in the papers by Myerson (1977), Bolger (1989), Feldman (1996), Albizuri, Arin and Rubio (2005), Pham Do and Norde (2007), and Macho-Stadler, Pérez-Castrillo and Wettstein (2007) (that we will refer to as $M P W$ ). These proposals satisfy the properties (axioms) of efficiency, anonymity (symmetry), linearity, and the "null" player property that states that players which have no effect on the outcome should neither receive nor pay anything. The particular definitions of these properties, at times together with some additional axioms, lead to different extensions of the Shapley value. ${ }^{1}$

In $M P W$ we strengthen the symmetry property by proposing the "strong symmetry axiom" capturing the idea that players with identical influence should receive the same outcome. We show that this axiom is equivalent to adopting an "average approach", which generates an attractive family of sharing methods (see Section 2).

In games without externalities, another useful approach to find sharing methods has been to take a constructive point of view. Harsanyi (1959) used the notion of dividends accruing to a player from the various coalitions he could participate in and showed that their sum yields the Shapley value. Maschler (1982) generalized this approach to a procedure through which the payoffs accruing to agents are still given by the Shapley value.

\footnotetext{
${ }^{1}$ de Clippel and Serrano (2008), rather than extend the Shapley axioms, focus on the implications of the marginality underlying the Shapley value, for games with externalities. McQuillin (2009) characterizes a value that simultaneously extends the Shapley value to games with externalities and situations where there is a prior coalition structure. Dutta, Ehlers and Kar (2008) adopt the potential approach to study and provide values for games with externalities.
} 
In this paper, we provide further support for the family of average values. We show that they can be constructed through the sharing of appropriately defined dividends. Furthermore, we show that this approach is flexible and can be easily adopted to generate non-symmetric values. Hence we provide a new family of weighted Shapley values for games with externalities and show that each one coincides with the weighted Shapley value of the average game.

\section{The environment and the average approach}

Cooperative environments with externalities are best described as games in partition function form (Thrall and Lucas, 1963). We denote by $N=\{1, \ldots, n\}$ the set of players. An embedded coalition is a pair $(S, P)$, where $S \subseteq N$ is a coalition and $P \ni S$ is a partition of $N$. An embedded coalition specifies the coalition as well as the organization of the rest of players. Also, we denote by $\mathcal{P}$ the set of all partitions of $N$ and by $E C L=$ $\{(S, P) \mid S \in P, P \in \mathcal{P}\}$ the set of embedded coalitions.

Let $(N, v)$ be a game in partition function form, where the characteristic function $v: E C L \rightarrow \mathbb{R}$ associates a real number with each embedded coalition. For each $(S, P) \in$ $E C L$, we interpret $v(S, P)$ as the worth of coalition $S$ when the players are organized according to the partition $P$. The partition $P$ is always taken to include the empty set $\varnothing$ and the characteristic function satisfies $v(\varnothing, P)=0$.

A sharing method, or a value, is a mapping $\varphi$ which associates with every game $(N, v)$ a vector in $\mathbb{R}^{n}$ that satisfies $\sum_{i \in N} \varphi_{i}(N, v)=v(N,\{N\})$. A value determines the payoffs for every player in the game and, by definition, it is always efficient since we assume that forming the grand coalition is the most efficient way of organizing the society.

To introduce the family of values proposed in $M P W$ using the average approach we present first the axioms that characterize it.

We say that player $i \in N$ is a null player in the game $(N, v)$ if $v(S, P)=v\left(S^{\prime}, P^{\prime}\right)$ for every $(S, P) \in E C L$ and for any embedded coalition $\left(S^{\prime}, P^{\prime}\right)$ that can be obtained from $(S, P)$ by changing the affiliation of player $i$.

The addition of two games $(N, v)$ and $\left(N, v^{\prime}\right)$ is defined as the game $\left(N, v+v^{\prime}\right)$ where $\left(v+v^{\prime}\right)(S, P) \equiv v(S, P)+v^{\prime}(S, P)$ for all $(S, P) \in E C L$. Similarly, given the game 
$(N, v)$ and the scalar $\lambda \in \mathbb{R}$, the game $(N, \lambda v)$ is defined by $(\lambda v)(S, P) \equiv \lambda v(S, P)$ for all $(S, P) \in E C L$.

For any permutation $\sigma$ of $N$, the $\sigma$ permutation of the game $(N, v)$, denoted by $(N, \sigma v)$ is defined by $(\sigma v)(S, P) \equiv v(\sigma S, \sigma P)$ for all $(S, P) \in E C L$.

The extension of Shapley (1953b)'s basic axioms proposed in $M P W$ for values in environments with externalities are:

1. Linearity: A value $\varphi$ satisfies the linearity axiom if:

1.1. For any two games $(N, v)$ and $\left(N, v^{\prime}\right), \varphi\left(N, v+v^{\prime}\right)=\varphi(N, v)+\varphi\left(N, v^{\prime}\right)$.

1.2. For any game $(N, v)$ and any scalar $\lambda \in \mathbb{R}, \varphi(N, \lambda v)=\lambda \varphi(N, v)$.

2. Symmetry: A value $\varphi$ satisfies the symmetry axiom if for any permutation $\sigma$ of $N$, $\varphi(N, \sigma v)=\sigma \varphi(N, v)$.

3. Null player: A value $\varphi$ satisfies the null player axiom if for any player $i$ which is a null player in the game $(N, v), \varphi_{i}(N, v)=0$.

In games with no externalities where the worth of any coalition $S$ does not depend on the organization of the other players, i.e., $v(S, P)=v\left(S, P^{\prime}\right)$ for every $S \subseteq N$ and $(S, P),\left(S, P^{\prime}\right) \in E C L$, these three basic axioms characterize a unique value (Shapley, 1953b). For expositional clarity, we denote by $(N, \widehat{v})$ a game with no externalities, where $\widehat{v}: 2^{N} \rightarrow \mathbb{R}$ is a function that gives the worth of each coalition. The Shapley value $\phi$ can be written as:

$$
\phi_{i}(N, \widehat{v})=\sum_{S \subseteq N} \beta_{i}(S, n) \widehat{v}(S) \text { for all } i \in N
$$

where we have denoted by $\beta_{i}(S, n)$ the following numbers:

$$
\beta_{i}(S, n)=\left\{\begin{array}{l}
\frac{(|S|-1) !(n-|S|) !}{n !} \text { for all } S \subseteq N, \text { if } i \in S \\
-\frac{|S| !(n-|S|-1) !}{n !} \text { for all } S \subseteq N \text {, if } i \in N \backslash S .
\end{array}\right.
$$

In games with externalities, the previous axioms are satisfied by a large set of values. In $M P W$ we propose an extension of the notion of symmetry also to the externalities created. The strong symmetry axiom strengthens the symmetry axiom by requiring that 
exchanging the names of the players inducing the same externality should also not affect the payoff of any player. To formally state the axiom, we denote by $\sigma_{S, P} P$, with $P \ni S$, a new partition with $S \in \sigma_{S, P} P$ resulting from a permutation of the set $N \backslash S$. Given an embedded coalition $(S, P)$, the $\sigma_{S, P}$ permutation of the game $(N, v)$ denoted by $\left(N, \sigma_{S, P} v\right)$ is defined by $\left(\sigma_{S, P} v\right)(S, P)=v\left(S, \sigma_{S, P} P\right),\left(\sigma_{S, P} v\right)\left(S, \sigma_{S, P} P\right)=v(S, P)$, and $\left(\sigma_{S, P} v\right)(R, Q)=v(R, Q)$ for all $(R, Q) \in E C L \backslash\left\{(S, P),\left(S, \sigma_{S, P} P\right)\right\}$.

2'. A value $\varphi$ satisfies the strong symmetry axiom if:

$2^{\prime} .1$. for any permutation $\sigma$ of $N, \varphi(N, \sigma v)=\sigma \varphi(N, v)$,

$2^{\prime} .2$. for any $(S, P) \in E C L$ and for any permutation $\sigma_{S, P}, \varphi\left(N, \sigma_{S, P} v\right)=\varphi(N, v)$.

As proven in $M P W$, any value $\varphi$ satisfying linearity and null player axioms also satisfies the strong symmetry axiom if and only if it can be constructed through the "average approach". This approach consists of, first constructing an average game $\left(N, \widehat{v}^{\alpha}\right)$ associated with $(N, v)$, by assigning to each coalition $S \subseteq N$ the average worth $\widehat{v}^{\alpha}(S) \equiv$ $\sum_{P \ni S, P \in \mathcal{P}} \alpha(S, P) v(S, P)$, with $\sum_{P \ni S, P \in \mathcal{P}} \alpha(S, P)=1$. We refer to $\alpha(S, P)$ as the "coefficient" of the partition $P$ in the computation of the value of coalition $S \in P$. Second, the average approach constructs a value $\varphi$ for the partition function game $(N, v)$ by taking the Shapley value of the game with no externalities $\left(N, \widehat{v}^{\alpha}\right)$. Therefore, if a value $\varphi$ is obtained through the average approach then, for all $i \in N$,

$$
\varphi_{i}(N, v)=\sum_{S \subseteq N} \beta_{i}(S, n) \widehat{v}^{\alpha}(S)=\sum_{(S, P) \in E C L} \alpha(S, P) \beta_{i}(S, n) v(S, P) .
$$

There are still a variety of values satisfying the requirements of linearity, strong symmetry, and null player, each value corresponding to a different averaging method. In $M P W$ we add a ${ }_{T}$ "similar influence axiom" to characterize a value with coefficients $\alpha(S, P)=\frac{T \in P \backslash S}{(n-|S|) !}$ (Feldman, 1996, also proposed but did not axiomatize this value). Other recent proposals also satisfy the average approach. The externality-free value studied and characterized by Pham Do and Norde (2007) and de Clippel and Serrano (2008) corresponds to the vector of coefficients $\alpha(S, P)=1$ if $P=\left\{S,\{j\}_{j \in N \backslash S}\right\}$ and $\alpha(S, P)=0$ otherwise; that is, only the partition where players outside $S$ form singleton coalitions is taken into account. On the other extreme, in his extension of the Shapley value McQuillin 
(2009) ends up proposing a value that corresponds to $\alpha(S, P)=1$ if $P=\{S, N \backslash S\}$ and $\alpha(S, P)=0$ otherwise; that is, the only important partition is the one where players outside $S$ form a single coalition. Finally, the proposal by Albizuri et al. (2005) corresponds to the Shapley value associated with the simple average: $\alpha(S, P)=\frac{1}{P(S)}$ where $P(S)=|\{(S, Q) /(S, Q) \in E C L\}|^{2}$

We denote by $\varphi^{\alpha}$ the value constructed through the average approach with a vector of coefficients $\alpha$. The analysis developed in the next section can be applied to any value $\varphi^{\alpha}$, in particular to those previously discussed.

\section{Dividends and weighted values in games with ex- ternalities}

In games with no externalities, the Shapley value can be characterized as the value that distributes the Harsanyi dividends of the game equally among the players in the corresponding coalitions. Harsanyi (1959) assumed that every coalition would negotiate a vector of "dividends" such that the sum of all coalitions' dividends vectors would be a feasible allocation for the grand coalition. Therefore, the dividends of a coalition $S$ are what is left after all proper subcoalitions of $S$ have received their corresponding dividends. More formally, the dividends $\Delta_{\widehat{v}}(S)$ of a game in characteristic form $(N, \widehat{v})$ are defined recursively: $\Delta_{\widehat{v}}(\varnothing)=0$ and $\Delta_{\widehat{v}}(S)=\widehat{v}(S)-\sum_{R \subsetneq S} \Delta_{\widehat{v}}(R)$ if $S \neq \varnothing$. Harsanyi (1959) showed that all the equal shares of the dividends a player is entitled to, sum up to his Shapley value:

$$
\phi_{i}(N, \widehat{v})=\sum_{S \ni i} \frac{1}{|S|} \Delta_{\widehat{v}}(S) \text { for all } i \in N .
$$

In a similar spirit, consider a game with externalities $(N, v)$ and a system of coefficients $\alpha$. We can obtain the dividends of a coalition $S$ when it is part of the partition $P$ taking into account that the dividends of subcoalitions of $S$ should matter only in proportion to

\footnotetext{
${ }^{2}$ The values proposed by Myerson (1977) and Bolger (1989) can not be constructed through the average approach. Some of the values proposed by Dutta, Ehlers and Kar (2008) can also be constructed through the average approach while others can not.
} 
its corresponding coefficients. Formally, we define the dividends inductively as follows:

$$
\begin{gathered}
\Delta_{v}^{\alpha}(\varnothing, P)=0 \\
\Delta_{v}^{\alpha}(S, P)=v(S, P)-\sum_{\substack{(R, Q) \in E C L \\
R \subsetneq S}} \alpha(R, Q) \Delta_{v}^{\alpha}(R, Q) \text { if } S \neq \varnothing .
\end{gathered}
$$

Proposition 1 provides an interpretation of any value $\varphi^{\alpha}$ in terms of dividends. Therefore, it is a result that can be applied, in particular, to the values studied by Feldman (1996) and MPW (2007); Albizuri et al. (2005); Pham Do and Norde (2007) and de Clippel and Serrano (2008); and McQuillin (2009).

Proposition 1 The value $\varphi^{\alpha}$ can be written as follows:

$$
\varphi_{i}^{\alpha}(N, v)=\sum_{\substack{(S, P) \in E C L \\ S \ni i}} \frac{1}{|S|} \alpha(S, P) \Delta_{v}^{\alpha}(S, P) .
$$

Proof. We denote by $\Delta_{\widehat{v}^{\alpha}}(S)$ the dividends of the average game $\left(N, \widehat{v}^{\alpha}\right)$ associated with the $(N, v)$. We prove by induction over the number of elements of $S$ that

$$
\Delta_{\widehat{v}^{\alpha}}(S)=\sum_{\substack{P \ni S \\ P \in \mathcal{P}}} \alpha(S, P) \Delta_{v}^{\alpha}(S, P) .
$$

The proof is immediate when $S=\varnothing$ (and it is also immediate when $|S|=1$ ). Assuming that the expression holds for any coalition $R$ with $|R|<|S|$, we can write

$$
\begin{aligned}
& \sum_{\substack{P \ni S \\
P \in \mathcal{P}}} \alpha(S, P) \Delta_{v}^{\alpha}(S, P)=\sum_{\substack{P \ni S \\
P \in \mathcal{P}}} \alpha(S, P)\left[v(S, P)-\sum_{\substack{(R, Q) \in E C L \\
R \subsetneq S}} \alpha(R, Q) \Delta_{v}^{\alpha}(R, Q)\right] \\
= & \sum_{\substack{P \ni S \\
P \in \mathcal{P}}} \alpha(S, P) v(S, P)-\sum_{\substack{P \ni S \\
P \in \mathcal{P}}} \alpha(S, P) \sum_{R \subsetneq S} \Delta_{\widehat{v}^{\alpha}}(R)=\widehat{v}^{\alpha}(S)-\sum_{R \subsetneq S} \Delta_{\widehat{v}^{\alpha}}(R)=\Delta_{\widehat{v}^{\alpha}}(S) .
\end{aligned}
$$

Since $\phi$ is the Shapley value of the (characteristic form game) $\widehat{v}^{\alpha}$, we can write $\phi_{i}(N, v)$ in terms of sum of dividends:

$$
\phi_{i}\left(N, \widehat{v}^{\alpha}\right)=\sum_{S \ni i} \frac{1}{|S|} \Delta_{\widehat{v}^{\alpha}}(S),
$$


therefore,

$$
\varphi_{i}^{\alpha}(N, v)=\phi_{i}\left(N, \widehat{v}^{\alpha}\right)=\sum_{S \ni i} \frac{1}{|S|} \sum_{\substack{P \ni S \\ P \in \mathcal{P}}} \alpha(S, P) \Delta_{v}^{\alpha}(S, P)=\sum_{\substack{(S, P) \in E C L \\ S \ni i}} \frac{1}{|S|} \alpha(S, P) \Delta_{v}^{\alpha}(S, P) .
$$

Maschler (1982) showed that the sharing method based on dividends was a special case of a procedure where a sequence of coalitions sequentially claim their worth. At each step the worth of one coalition is equally shared by its members. The process then generates a new game where the worth of each coalition containing the sharing coalition is reduced by the worth of the sharing coalition, whereas the worth of other coalitions is not altered. The next coalition in the sequence then proceeds to share its worth. This process ends when all coalitions arrive at zero worth. It was shown that this process must end, and the sum of payoffs accruing to each player is his Shapley value. The value $\varphi^{\alpha}$ can be arrived at via these more general procedures as well if the proportion $\alpha(S, P)$ of the dividend associated with each embedded coalition $(S, P)$ is assigned to each player $i \in S$ and the coefficient $\alpha(S, P)$ is also used to reduce the worth of each coalition containing $S$.

We can use the dividends we have constructed to derive a family of weighted values for games with externalities, similarly to the Shapley (1953b) construction for games without externalities. We define, for a given vector of weights $w \in \mathbb{R}^{n}$, with $w_{i}>0$ for $i \in N$, (and for each vector of coefficients $\alpha$ ) a weighted Shapley value for games with externalities:

$$
\varphi_{i}^{\alpha w}(N, v)=\sum_{(S, P) \in E C L} \frac{w_{i}}{w_{S}} \alpha(S, P) \Delta_{v}^{\alpha}(S, P) .
$$

Note that we keep the symmetric treatment of externalities (since the coefficients $\alpha(S, P)$ are anonymous) while allowing for different bargaining powers when sharing the dividends.

It follows easily that there is a relationship between the weighted Shapley value for games with externalities that we have defined through the dividends and the weighted Shapley value of the corresponding average game $\left(N, \widehat{v}^{\alpha}\right)$. We state this result in the next proposition:

Proposition 2 The value $\varphi^{\alpha w}$ can be written as follows:

$$
\varphi_{i}^{\alpha w}(N, v)=\phi_{i}^{w}\left(N, \widehat{v}^{\alpha}\right)
$$


Proof. We write the value $\phi^{w}$ :

$$
\phi_{i}^{w}\left(N, \widehat{v}^{\alpha}\right)=\sum_{\substack{S \subseteq N \\ S \ni i}} \frac{w_{i}}{w_{S}} \Delta_{\widehat{v}^{\alpha}}(S)=\sum_{\substack{S \subseteq N \\ S \ni i}} \frac{w_{i}}{w_{S}} \sum_{\substack{P \ni S \\ P \in \mathcal{P}}} \alpha(S, P) \Delta_{v}^{\alpha}(S, P)
$$

following the proof of Proposition 1. Therefore,

$$
\phi_{i}^{w}\left(N, \widehat{v}^{\alpha}\right)=\sum_{\substack{S \subseteq N \\ S \ni i}} \sum_{\substack{P \ni S \\ P \in \mathcal{P}}} \frac{w_{i}}{w_{S}} \alpha(S, P) \Delta_{v}^{\alpha}(S, P)=\sum_{\substack{(S, P) \in E C L \\ S \ni i}} \frac{w_{i}}{w_{S}} \alpha(S, P) \Delta_{v}^{\alpha}(S, P)=\varphi_{i}^{\alpha w}(N, v)
$$

Finally, we note that the value $\varphi^{\alpha w}(N, v)$ can be implemented via bidding mechanisms in the same spirit as the ones that appear in Macho-Stadler et al. (2006), for any system of non-negative coefficients, i.e., $a(S, P) \geq 0$ for all $(S, P) \in E C L$, as it is shown in Macho-Stadler et al. (2008).

\section{References}

[1] Albizuri MJ, Arin J, Rubio J (2005) An axiom system for a value for games in partition function form. Int Game Theory R 7:63-73.

[2] Bolger EM (1989) A set of axioms for a value for partition function games. Int J Game Theory 18:37-44.

[3] de Clippel G, Serrano R (2008) Marginal contributions and externalities in the value. Econometrica 76:1413-1436.

[4] Dutta B, Ehlers L, Kar A (2008) Externalities, potential, value and consistency. WP Univ Montréal.

[5] Feldman BE (1996) Bargaining, coalition formation, and value. Ph.D. thesis, State Univ of New York at Stony Brook.

[6] Harsanyi JC (1959) A bargaining model for the cooperative $n$-person game. In Contributions to the Theory of Games IV, ed by Tucker AW, Luce RD, 325-355, Princeton Un Press, Princeton. 
[7] Macho-Stadler I, Pérez-Castrillo D, Wettstein D (2006) Efficient bidding with externalities. Games Econ Behaviour 57:304-320.

[8] Macho-Stadler I, Pérez-Castrillo D, Wettstein D (2007) Sharing the surplus: A just and efficient proposal for environments with externalities. J Econ Theory 135:339356.

[9] Macho-Stadler I, Pérez-Castrillo D, Wettstein D (2008) Dividends and weighted values in games with externalities. Barcelona GSE Research Network WP 366.

[10] Maschler, M (1982) The worth of a cooperative enterprise to each member in Games, Economic Dynamics and Time Series Analysis, ed. by Deistler M, Furst F, Schwodiaur G, Vienna-Wurzburg, Physica-Verlag.

[11] McQuillin, B (2009) The extended and generalized Shapley value: Simultaneous consideration of coalitional externalities and coalitional structure. J Econ Theory 144:696-721.

[12] Myerson RB (1977) Values of games in partition function form. Int J Game Theory 6:23-31.

[13] Pham Do KH, Norde H (2007) The Shapley value for partition function form games. Int Game Theory R 9:353-360.

[14] Shapley LS (1953a) A value for $n$-person games. In Kuhn, H.W., Tucker, A.W. (Eds.), Contributions to the theory of games II, 307-317. Princeton Un Press, Princeton.

[15] Shapley LS (1953b) Additive and non-Additive set functions. Ph.D. thesis, Princeton Univ, Princeton. 